\title{
Kernos
}

Revue internationale et pluridisciplinaire de religion grecque antique

$6 \mid 1993$

Varia

\section{Orthia et la flagellation des éphèbes spartiates}

Un souvenir chimérique de sacrifice humain

\section{Pierre Bonnechere}

\section{Q OpenEdition \\ Journals}

\section{Édition électronique}

URL : http://journals.openedition.org/kernos/533

DOI : $10.4000 /$ kernos.533

ISSN : 2034-7871

Éditeur

Centre international d'étude de la religion grecque antique

\section{Édition imprimée}

Date de publication : 1 janvier 1993

Pagination : 11-22

ISSN : 0776-3824

\section{Référence électronique}

Pierre Bonnechere, «Orthia et la flagellation des éphèbes spartiates », Kernos [En ligne], 6| 1993, mis en ligne le 07 avril 2011, consulté le 01 mai 2019. URL : http://journals.openedition.org/kernos/533 ; DOI : 10.4000/kernos.533 
Kernos, 6 (1993), p. 11-22.

\section{ORTHIA ET LA FLAGELLATION DES ÉPHÈBES SPARTIATES. UN SOUVENIR CHIMÉRIQUE DE SACRIFICE HUMMAIN}

Sur les aspects insolites que le culte spartiate d'Orthia ${ }^{1}$ drainait dans son sillage ${ }^{2}$ plane une énigmatique rumeur de sacrifices humains à propos de la flagellation des jeunes Spartiates sur le $\beta \omega \mu$ ś de la cruelle divinité.

Le texte de Pausanias (III, 16, 9-10), indéniablement ciselé avec une précision démoniaque, affiche, semble-t-il, une grande cohérence interne, qui a le mérite de s'adapter à une des principales théories sur la nature des cérémonies religieuses qui prenaient place dans le sanctuaire de la déesse, à savoir le passage des adolescents dans la société des adultes ${ }^{3}$.

1 Le nom de la divinité est Orthia, et son assimilation avec Artémis n'advient que sous les Flaviens : voir A.M. WooDWARD, Inscriptions, in The Sanctuary of Artemis Orthia at Sparta, Excavated and Described by Members of the British School at Athens, 1906-1910, éd. R.M. DAwkINs, Londres, 1929 (JHS, suppl. 5), p. 293 [ci-après cité AO]; H.J. Rose, The Cult of Artemis Orthia, in $A O$ (cité n. 1), p. 401, n. 11. Orthia semble s'identifier pleinement à la IIótvıa onpâ, au même titre qu'Artémis : Ibidem, p. 401-403; K.T.M. CHRImes, Ancient Sparta. A Re-Examination of the Evidence, Manchester, $1952^{2}$, p. 248-259; A. BRELICH, Paides e Parthenoi, Rome, 1969 (Incunabula Graeca, 36), p. 130-131.

2 Voir essentiellement A. B RELICH (cité n. 1), p. 126-140; C. CALAME, Les chceurs de jeunes filles en Grèce archaïque, t. 1, Rome, 1977, p. 276-297. F. FronTisI Ducroux, La bomolochia : autour de l'embuscade à l'autel, in RCGO, 2, Naples, 1984 (Cahiers du Centre Jean Bérard, 9), p. 29-49; J.-P. VERNANT, Une divinité des marges : Artémis Orthia, in RCGO, 2, Naples, 1984, p. 13-27.

3 Sur ces notions désormais acquises de «passage» au sein de la civilisation grecque, voir essentiellement H. JEANMAIRE, Couroi et Courètes. Essai sur l'éducation spartiate et les rites d'adolescence dans l'antiquité hellenique, Lille, 1939; A. Brelich, Paides et parthenoi (cité n. 1); C. CAlame, Les chours de jeunes filles (cité n. 2); B. SERGENT, L'homosexualité dans la mythologie grecque, Paris, 1984 (Bibliothèque Historique); Les rites d'initiation. Actes du colloque de Liège et de Louvain-la-Neuve, 20-21 novembre 1984, éds J. RIES et H. Limet, Louvain-la-Neuve, 1986 (Homo Religiosus, 13); B. SERGENT, L'homosexualité initiatique dans l'Europe ancienne, Paris, 1986 (Bibliothèque Historique); P. BRulé, La fille d'Athènes. La religion des filles à Athènes à l'époque classique. Mythes, cultes, sociétés, Paris, 1987 (Annales littéraires de 


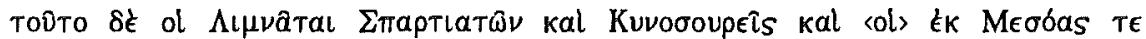

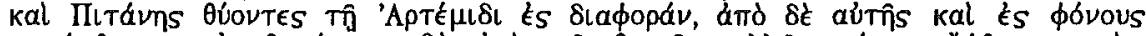

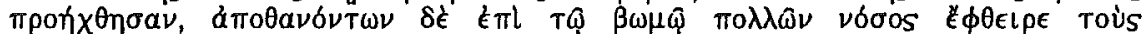

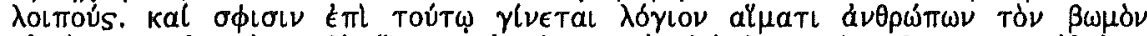

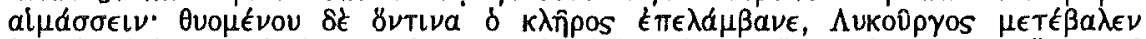

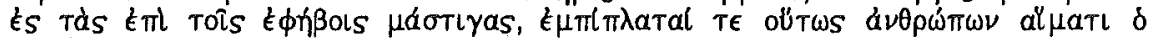

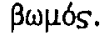

En second lieu, les Spartiates de Limnatis et de Cynosouries, et ceux de Mésoa et de Pitané, alors qu'ils sacrifiaient à Artémis, connurent un désaccord à la suite duquel ils en vinrent à faire couler le sang; après que beaucoup eurent succombé à l'autel, un fléau s'abattit sur les autres. Et à ce propos un oracle leur fut rendu qui leur enjoignait d'arroser l'autel de sang humain : aussi dorénavant celui que le sort avait désigné était sacrifié, mais Lycurgue transforma la coutume en la flagellation des éphèbes, de sorte que l'autel soit de même couvert de sang humain.

C'est là, selon le Périégète, l'origine de cette obligation incombant à tout Spartiate, dans sa jeunesse, de se soumettre avec une sérénité endurante aux coups des fouets dans le cadre de ce festival religieux hors du commun. Les plus nobles et les plus beaux se faisaient un honneur de briller particulièrement lors de l'épreuve annuelle, et tous forçaient l'admiration tant des familles que des curieux attirés par le spectacle ${ }^{4}$. Le vainqueur de ce rituel-concours gagnait le titre de $\beta \omega \mu o \nu(\epsilon)\left(k n s^{5}\right.$, jouissait d'une estime grandie et parfois bénéficiait d'une statue $^{6}$ : son exploit, il est vrai, comportait d'importants risques, car la perte de sang pouvait atteindre des proportions critiques et le décès d'un des concurrents, battus nus sans répit, advenait de temps à autre encore que jamais il n'ait été souhaité ${ }^{7}$.

l'Université de Besançon, 363); K. Dowden, Death and the Maiden. Girl's Initiation Rites in Greek Mythology, Londres-New York, 1989.

4 CicÉron, Tusculanes, XIV, 34; LUCIEN, Anacharsis, 38; PAUSANIAS, III, 16, 7-11; Philostrate, Vie d'Apollonius de Tyane, VI, 20; Plutarque, Anciennes coutumes des Spartiates, 40 (Mor., 239d); S EXTUS EMPIRICUS, Grandes lignes du Pyrrhonisme, III, 208.

$5 I G, \mathrm{~V}, 1,554 ; 652 ; 653$ A-B (= A.M. WoOdWARD [cité n.1 ]), nº 142-143); 654; A.M.

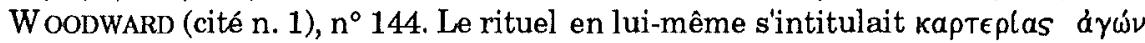
( IG, V, 1, 290 et 653 A [ = A.M. WoODWARD, (cité n. 1), n 37 et 142]); voir encore Platon, Lois, I, 633b · kaptephofls, et Plutarque, Anciennes coutumes des

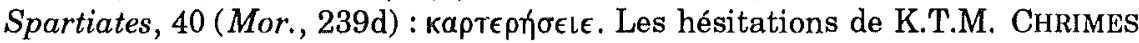
(citée n. 1), p. 135-136, sont peu convaincantes.

6 Les n 142-144 de A.M. Woodward (cité n. 1) sont des bases de statues. Selon LUCIEN (Anacharsis, 38), ces statues auraient été élevées aux frais de l'État.

7 Voir Cicḱron, Tusculanes, XIV, 34; Plutarque, Anciennes coutumes des Spartiates, 40 (Mor., 239d); ID., Lycurgue, XVIII, 1-2. LuCIEN (Anacharsis, 38) 
«Ethnologiquement,» - conclut Claude Calame ${ }^{8}$, - «le rite de la flagellation au sanctuaire d'Artémis Orthia se définit donc comme un rite d'initiation, et plus précisément un rite d'initiation tribale. En tant que tel, il comporte la structure à trois phases caractéristique de tous les rites de passage. Entre la séparation de l'ordre ancien (enfance) et la réintégration à l'ordre nouveau (âge adulte), il représente, à côté d'autres connotations, le stade de la mort, de la ségrégation, de l'immersion dans le chaos", pour reprendre les termes d'Eliade".

Et les raisons ne manquent pas pour accréditer cette séduisante hypothèse : la mort initiatique et donc symbolique par fustigation et effusion conséquente de sang est explicitement exprimée dans l'aition de Pausanias, puisque la flagellation y équivaut au sacrifice humain des origines, auquel échappent désormais les éphèbes des temps historiques. Les participants sont nus, et l'on sait à quel point la nudité est importante en ces circonstances, tandis que l'échappatoire qui permet l'abolition des immolations pourrait être comparé à l'ámám dont doivent faire preuve les novices avant d'accéder au monde des adultes où la ruse doit s'effacer devant la force et l'esprit hoplitiques. On citerait même volontiers un parallèle attique, celui de la retraite artémisiaque des petites athéniennes qui, au noviciat de la vie civique, font les ourses pour la déesse : le mythe étiologique de la cérémonie expliquait qu'une fille de citoyen devait expier de sa vie le meurtre sacré de l'ourse de la divinité; son père cependant, nommé d'ailleurs Embaros, avait alors eu recours à une ruse par laquelle il immolait à la place de sa fille une chèvre pareillement vêtue ${ }^{9}$.

Le passage du Périégète a été récemment invoqué par Jane B. Carter dans une perspective tout autre, celle de démontrer la fondation du culte d'Orthia à Sparte par des Phéniciens, adorateurs d'Asherah-Tanit, déesse qui offrirait de sérieuses ressemblances avec la divinité

parle de très nombreux morts, mais le sarcasme dont tout le dialogue est empreint laisse transparaître une nette exagération. Philostrate (Vie d'Apollonius de Tyane, VI, 20) précise que le but de la cérémonie était justement d'épargner aux éphèbes la mort sacrificielle qui jadis leur était promise.

8 C. CALAME (cité n. 2), p. 279; voir aussi p. 291.

9 Sur les cultes artémisiaques de l'Attique, et leurs relations avec les cérémonies initiatiques présidant à l'intégration des filles et garçons d'Athènes dans la société adulte, voir en dernier lieu P. BRULÉ (cité n. 3), passim et K. DowDEN (cité n. 3), p. 19-48. 
spartiate $^{10}$. Eu égard à mes connaissances trop peu approfondies de la religion punique, il ne m'appartient pas de juger cette hypothèse, en dépit de son caractère apparemment très hasardeux ${ }^{11}$. Cependant, pour asseoir sa démonstration, Mme Carter en vient à comparer les sacrifices humains offerts par les Carthaginois lors du rituel du Molk, à Tanit et à Baal, au souvenir d'immolations humaines dans le culte d'Orthia : celles-ci, à l'origine, auraient été accomplies par les adorateurs phéniciens de ce culte nouvellement fondé et encore proche de ses origines : "The whipping of ephebes on Ortheia's altar could be a substitution for the original rite of child sacrifice introduced by the Phoenicians» 12 .

Ces deux utilisations du texte du Périégète se fondent néanmoins sur une analyse trop rapide du corpus de documents que l'on peut réunir sur cette particularité du culte spartiate, et une révision de celui-là, pourtant connu depuis de longues années, ne sera dès lors pas inutile ${ }^{13}$.

10 J.B. CARTER, The Masks of Ortheia, in AJA, 91 (1987), p. 355-383, et en particulier p. 378-383.

11 On aurait pu s'attendre au moins à ce que l'auteur souligne certains éléments semblables dans les deux types allégués de sacrifice humain. Or, sa comparaison semble reposer sur la seule existence d'immolations humaines dans les cultes d'Orthia et de Tanit et Baal. Une tradition de sacrifice humain, cependant, n'est pas l'autre, et l'on regrettera tout autant que Mme Carter ne se soit pas arrêtée aux possibilités d'explication des sacrifices spartiates par comparaison avec le très riche répertoire des traditions de sacrifices humains en Grèce propre. La tentation d'établir une filiation entre les sacrifices humains grecs et les sacrifices humains carthaginois (d'ailleurs loin d'être aussi certains qu'on ne le dit habituellement) n'est pas nouvelle : on verra V. BÉRARD, $D e$ l'origine des cultes arcadiens, Paris, 1894, passim; L.R. FARNELL, The Cults of the Greek States, t. 1, Oxford, 1896, p. 201-204; J. Herbillon, Les cultes de Patras, Baltimore, 1929, p. 46-49; R. ReBUfFAT, Le sacrifice du fils de Créon dans les "Phéniciennes» d'Euripide, in REA, 74 (1972), p. 14-31, etc.

12 J.B. CARTER (citée n. 10), p. 381 et n. 165.

13 Pour exemple, voir R. FlaCelitre, Sur quelques passages des Vies de Plutarque. II. Lycurgue-Numa, in REG, 61 (1948), p. 398-400; H. JEANMAIRE, (cité n. 3), p. 518; H.I. Marrou, Histoire de l'éducation dans l'Antiquité. I. Le 
D'abord, la fustigation exposée par Pausanias n'est ainsi décrite pour la première fois que par Cicéron et, telle quelle, appartient à la période romaine. Toutes les inscriptions désignant des vainqueurs de l'épreuve sont d'époque impériale, au même titre que l'aition du Périégète ${ }^{14}$. De plus, ce genre de cérémonie, où la résistance physique est reine, s'intègre bien à ce vaste mouvement de "retour aux sources» que vécut la Sparte des premiers siècles de notre ère ${ }^{15}$, et qui incita la cité à édifier pour cette seule fête, vers 250 après J.-C., un spacieux théâtre destiné à accueillir la foule toujours grandissante des amateurs de sensations fortes ${ }^{16}$.

Or Xénophon, témoin privilégié des institutions classiques laconiennes, avait également connaissance de la fustigation des éphèbes, mais il la présente sous l'aspect d'une joute rituelle entre deux groupes d'adolescents, le premier s'essayant à dérober avec précision et vivacité des fromages disposés sur l'autel d'Orthia, le second à s'y opposer au moyen de fouets ${ }^{17}$.

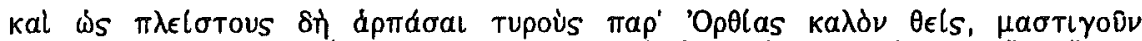

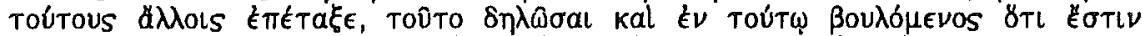

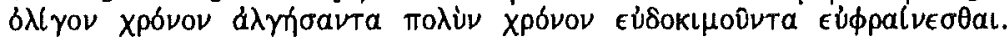

monde grec, Paris, $1965^{6}$, p. 53-54 et 353, n. 31. P. Roussel, Sparte, Paris, $1947^{2}$, p. 152-153.

14 Aucune inscription ayant trait à la flagellation pure et simple, telle qu'elle existait sous la domination de Rome, ne remonte au-delà de ca 100 ap. J.-C., en dépit des paragraphes allusifs d'A. B RELICH (cité n. 1, p. 133-134; 174-175; 192) et de C. CALAME (cité n. 2, p. 296), qui laissent sous-entendre que des inscriptions agonistiques remonteraient au IVe siècle av. J.-C. (sans toutefois les préciser). Au total, sur une dizaine d'inscriptions remontant au-delà de ca 50

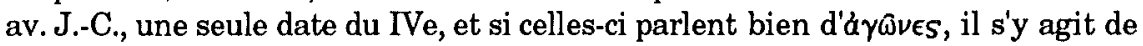

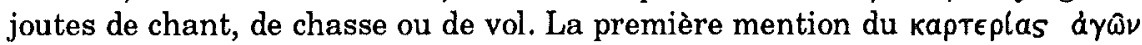
(A.M. WoODWARD [cité n. 1], $\mathrm{n}^{\circ}$ 37) date de ca 100 ap. J.-C., et la seconde (A.M. WOODWARD [cité n. 1], $\mathrm{n}^{\circ}$ 142) de ca 200. Le titre de $\beta \omega \mu \nu \nu(\epsilon)$ lkns se rencontre

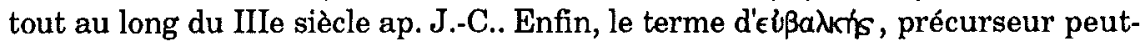

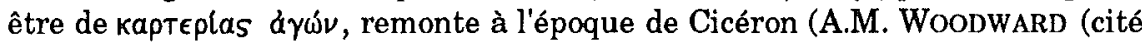
n. 1$), \mathrm{n}^{\circ} 16 ; 18 ; 83$ ).

15 Voir l'avis unanime des modernes : H. Jeanmaire (cité n. 3), p. 516; H.I. Marrou (cité n. 13), p. 53; H.J. Rose (cité n. 1), p. 405; P. Roussel (cité n. 13), p. 153.

16 R.M. DAwKINS, The History of the Sanctuary, in $A O$ (cité n. 1), p. 37-47.

17 Constitution de Sparte, II, 9. Signalons que même la découverte d'une inscription mentionnant un $\beta \omega \mu \nu \nu(\epsilon)(k n s$ datant de l'époque classique ne serait pas capitale, car le titre pourrait désigner, à l'origine, non celui ayant enduré le plus de coups, mais le meilleur voleur de fromages. 
$\Pi$ (Lycurgue) institua donc comme un bien de voler un maximum de fromages de l'autel d'Orthia, et prescrivit à d'autres de fouetter les voleurs, désireux de montrer en cela qu'en souffrant pour un court laps de temps, on pouvait être charmé longtemps d'une excellente renommée.

L'allusion peut sembler évanescente et beaucoup d'éditeurs de la Constitution de Sparte placent le passage entre crochets, comme s'il s'agissait d'une interpolation, en témoignant de leur embarras à rattacher cet épisode à la description détaillée de Pausanias. La dissemblance avec le témoignage du Périégète et des autres auteurs d'époque romaine ne doit pas suffire cependant pas à jeter l'anathème sur l'extrait de Xénophon : ainsi de part et d'autre il est question du culte d'Orthia et de flagellation d'éphèbes, et il semble inacceptable de supposer deux rituels distincts de flagellation dans l'orbite du même sanctuaire d'Artémis. Et ce d'autant moins qu'un texte de Plutarque permet de réunir les données divergentes de l'exilé athénien et des auteurs postclassiques: le biographe décrit ainsi la conduite du roi de Sparte à Platées, alors que l'engagement du combat contre les Perses de Mardonios est imminent ${ }^{18}$ :

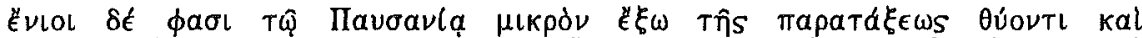

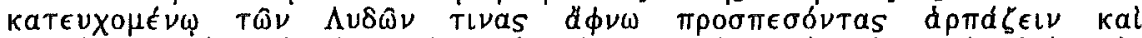

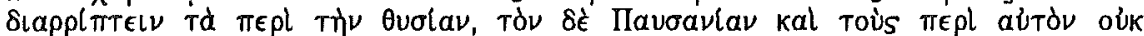

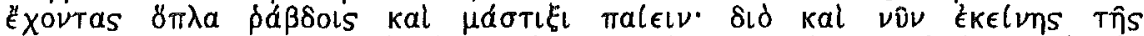

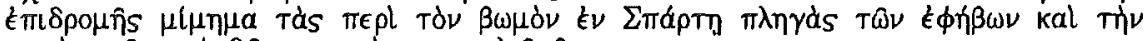

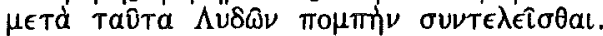

Selon quelques auteurs, au moment où Pausanias sacrifiait et priait un peu en dehors de la ligne, une bande de Lydiens, tombant soudain sur lui, arracha et dispersa tout ce qui servait au sacrifice; Pausanias et ceux qui l'entouraient, n'ayant pas d'armes, les frappèrent avec des bâtons et des fouets. C'est pour cela qu'aujourd'hui encore, en imitation de cette attaque, on frappe à Sparte les éphèbes autour de l'autel et qu'on fait ensuite la procession des Lydiens 19.

L'anecdote, de toute évidence, est censée fournir l'explication de la flagellation d'endurance, la seule que Plutarque connaisse et cite : "c'est pour cela qu'aujourd'hui encore..."; mais celle-ci se rapporte davantage à la cérémonie de la fin du Ve siècle avant J.-C., par ses

18 Aristide, XVII, 10. Ni le consciencieux A. BRELICH (cité n. 1, p. 135-136; ID, Symbol of a Symbol, in Myths and Symbols. Studies in Honour of Mircea Eliade, Chicago, 1969, p. 201), ni l'excellent C. CALAME (cité n. 2, p. 296-297), ni J.B. CARTER (citée n. 10, p. 381) ne se sont préoccupés des aitia de Plutarque et Philostrate, tout en mettant en exergue l'aition du Périégète, particulièrement intéressant pour leurs propos.

19 Édition et traduction de R. Flacelière et E. Chambry (CUF). 
allusions à la lutte entre deux bandes, dont l'une tente d'empêcher, et à coups de fouets, la seconde d'approcher de l'autel. Plutarque, du reste, avait eu vent de l'algarade aux fromages, car dans la Vie de Lycurgue, il avait logiquement placé l'épisode de l'autel d'Orthia juste après avoir abordé la question des vols que la loi spartiate exigeait des enfants ${ }^{20}$.

Cette constatation sans appel permet donc d'affirmer que Xénophon et les auteurs plus tardifs parlent de la même cérémonie, et du même coup que la célébration d'époque romaine n'était qu'un avatar de l'empoignade aux fouets décrite dans la Constitution de Sparte. C'est donc que le rituel avait subi une nette évolution à la période hellénistique : que l'appartenance de la sanglante épreuve de pure endurance au noyau initial du festival ne peut donc plus être valablement défendue ${ }^{21}$.

De surcroît, l'aition de Pausanias, capital pour l'interprétation "initiatique" du rite ainsi que pour la comparaison avec le domaine sémitique, n'est pas unique : non seulement il est concurrencé par celui de Plutarque, mais aussi par un second, œuvre de Philostrate, tous deux légèrement plus anciens et fréquemment passés sous silence.

Philostrate s'entend avec le Périégète pour identifier la statue d'Orthia à l'image taurique d'Artémis ramenée de Scythie par Oreste : l'ancienneté de la divinité spartiate est donc rattachée à un mythe de facture relativement jeune, datant selon toute probabilité du Ve siècle avant J.-C. ${ }^{22}$; et l'on sait que plusieurs cultes ont revendiqué tardivement la possession du fameux ḱóavov parce qu'une partie de leur rituel apparaissait - à l'origine du moins - trop cruelle ou étrangère pour n'avoir pas été importée de ces contrées sauvages dont la Tauride était emblématique $^{23}$. Si Pausanias relate ensuite les sacrifices humains en

\section{Lycurgue, XVII-XVIII, 1-2.}

21 Malgré la faiblesse d'un tel argument, on s'expliquerait mal également, si Sparte avait depuis toujours connu pareille flagellation, le silence des Athéniens, à tout moment prêts à ridiculiser leurs ennemis héréditaires, auxquels Lucien (Anacharsis, 38), avec l'humour qu'on lui connait, conseille en l'occurrence quelques graines d'ellébore.

22 Philostrate, Vie d'Apollonius de Tyane, VI, 20 (Vaticani Mythographi, I, 173). Voir C. RoBERT, Griechische Mythologie, I, Berlin, 1920, p. 312-315, suivi par H. G RÉGOIRE (éd.), Iphigénie en Tauride, Paris, 1925, p. 110 (CUF).

23 Voir l'excellent article de F. GRAF, Das Götterbild aus dem T'aurerland, in AW, 10 (1979), p. 33-41, et W. Burkert, Greeh Religion. Archaic and Classical, Oxford, 1985, p. 152. 
relation avec une banale affaire de fléau, Philostrate fait simplement référence à ceux dont la divinité était honorée en Tauride même, tandis que tous deux rapportent aux Spartiates l'initiative de leur avoir substitué un fouettement à sang.

Peu importent d'ailleurs les divergences entre ces deux aitia : focalisés uniquement sur la flagellation, ils ne pourraient expliquer que la manifestation d'époque romaine; ils sont inaptes l'un autant que l'autre à rendre compte de la cérémonie relatée par l'exilé athénien, puisqu'on n'y trouve trace ni de lutte ni de vol, pour cette simple raison sans doute qu'au moment de leur élaboration, ces deux composantes avaient déjà disparu du rituel. Aucune présomption d'ancienneté ne peut donc leur être accordée. Il en va autrement de l'aition présenté par Plutarque, limité toutefois au terminus post quem tout théorique de 479 avant J.-C.

Au total, sans nullement récuser les étroites relations entre le culte d'Orthia et les rites de passage, d'ailleurs intimement fondus dans tout le système éducatif spartiate, il est inadmissible d'accorder à la fustigation tardive, sur base d'un aition non moins tardif, une haute antiquité, ou encore de la présenter comme représentatrice du plus pur rite d'initiation tribale.

La manifestation classique n'en apparaît pas moins comme un rite d'initiation, précédé d'une retraite à la campagne et suivi d'une procession, dite des Lydiens, elle aussi rattachée à l'aition de Plutarque ${ }^{24}$, en laquelle on a coutume de reconnaître la présentation, devant la communauté adulte, des éphèbes parvenus au terme de leur parcours préparatoire ${ }^{25}$. Cependant le caractère de mort rituelle assigné à la

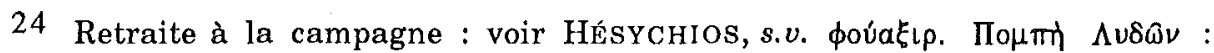
PluTARQUE, Aristide, XVII, 10 (l'association des adolescent(e)s et des Lydiens existe ailleurs : ARISTOPHANE, Nuées, 599-600; AUTOCRATÈs, fr. 1 Kassel-Austin; Ion de Chios, fr. 22 Snell; voir C. Calame [cité n. 2], p. 182-183). Cette procession, dont nous ignorons tout, avait incité H. JEANMAIRE (cité n. 3, p. 518519) à corriger le terme de Plutarque, en émendant $\Lambda \nu \delta \omega \nu$ en $\Lambda u ́ k \omega \nu$, et en en faisant la procession des loups, animaux dont il s'est ingénié à montrer l'importance capitale dans le système éducatif spartiate. Malgré son caractère judicieux, cette correction doit céder le pas à une lectio difficilior, d'autant plus que cette dernière est parfaitement explicable dans le contexte rituel spartiate.

25 Voir W. Burkert, Demaratos, Astrabakos und Herakles. Königsmythos und Politik zur Zeit der Perserkriege (Herodot, 6, 67-69), in $M H, 22$ (1965), p. 166 177; C. Calame (cité n. 2), p. 280, n. 211; F. GraF (cité n. 23), p. 41; H. JEANMAIRe (cité n. 3), p. 518-519 (quelque peu réticent). La collection unique de masques trouvée dans les couches archéologiques du sanctuaire (et éditée 
flagellation doit composer avec ce thème central qu'est le vol de fromages, tout en étant déforcé par l'aition de Plutarque sans plus pouvoir s'appuyer sur celui du Périégète : ce caractère de mort rituelle ne saurait donc plus être qualifié de certitude inébranlable comme il l'a été par le passé ${ }^{26}$; il apparaît au contraire peu crédible. Les modalités de «l'initiation spartiate» sont complexes et mal connues, et la mort symbolique (à supposer qu'on puisse la repérer) pourrait être cherchée dans d'autres manifestations; pour ne fournir qu'un exemple, au Platanistas, deux groupes d'éphèbes s'affrontent jusqu'à ce qu'une des bandes soit rejetée dans l'eau, et l'immersion, elle aussi, est souvent considérée comme simulacre du trépas rituel : la prudence reste donc de rigueur.

par G. Dickins, The Masks, in $A O$ [cité n. 1], p. 163-186) semble faire partie intégrante de rituels de travestissement propres aux cérémonies de passage : voir maintenant J.B. CARTer, Masks and Poetry in Early Sparta, in Early Greek Cult Practice. Proceedings of the Fifth International Symposium at the Swedish Institute at Athens, 26-29 June 1986, Stockholm, 1988 (ActaAth, 38), p. 89-98 et EAD. (citée n. 10), p. 355-374, pour une intéressante comparaison entre les masques spartiates et certains masques puniques. Les festivités dans le culte d'Orthia requéraient également d'importants chœurs de jeunes filles (C. CALAME, cité n. 2, p. 281-289). On verra encore Le masque de théâtre dans l'Antiquité classique, Arles, 1986, p. 3-4; H. JEANMaIRE (cité n. 3), p. 513-523; J.P. VERNANT (cité n. 2), p. 13-27.

26 A. Brelich (cité n. 1), p. 136; 154, suivi par C. CALAME (cité n. 2, p. 279; 291), par D. Hughes (Human Sacrifice in Ancient Greece, Londres-New York, 1991 p. 80-81), par M. MAzzenzio (La festa di Artemis Triklaria e Dionysos Aisymnetes a Patrai, in SMSR, 39 [1968], p. 100-132) et avec réserves par F. DE PoligNaC (La naissance de la cité grecque; cultes, espace et société; VIIIe-VIIe siècles avant J. .C., Paris, 1984 [ Textes à l'appui. Histoire classique], p. 68). L.R. FARNELL (cité n. 11), II, Oxford, 1896, p. 439, tout en reconnaissant le caractère initiatique du rituel, estimait que l'effusion de sang sur l'autel renforçait le lien entre l'initié et la communauté adulte, regroupée sous l'égide de la déesse (et à sa suite H. Michell, Sparte et les Spartiates, trad. A. Coeuroy, Paris, 1953, p. 139-140), mais cette hypothèse suppose que le sang ruisselant fut dès l'origine l'élément prédominant de la cérémonie. Peu défendable seule, la fustigation par la Lebensrute a été proposée par A. THOMSEN (Orthia, in ARW, 9 [1906], p. 397-416) et acceptée en général par M.P. Nilsson (Griechische Feste von religiösen Bedeutung, Leipzig, 1906, p. 190-196) et par S. REINACH (La flagellation rituelle, in Cultes, Mythes et Religions, 1, Paris, 1922 ${ }^{3}$, p. 173-183). En aucune façon les fustigés ne peuvent être assimilés aux boucs émissaires athéniens (comme le voulait K.T.M. Chrimes (citée n. 1), p. 260-265, ni la flagellation à un simple fertility charm (L.B. LAWLER, The Dance in Ancient Greece, Londres, 1964, p. 26). 
De même, l'idée de la substitution d'une fustigation à des sacrifices humains ne saurait être tenue pour ancienne : elle ne peut en aucun cas dater du temps de Xénophon et de la première phase connue de l'histoire du rituel, car elle aurait constitué un aition qui n'aurait en rien expliqué le pourquoi de la totalité des éléments constitutifs de la cérémonie. En d'autres termes, elle n'a pu prendre forme que lorsque l'autel ensanglanté d'Orthia fut devenu l'unique intérêt d'un rituel à bout de souffle ${ }^{27}$. La signification de la manifestation s'étant peu à peu transformée jusqu'à devenir une probation de résistance à la douleur, il fallut en justifier l'existence face à des explications devenues désuètes, en comparaison peut-être avec d'autres cultes aux pratiques similaires en cruauté apparente.

Puisque l'homme, par nature, répugne à faire et à voir couler le sang, l'horreur que devait susciter la mort de certains participants en tant que partie intégrante du contexte religieux, associée au spectacle impressionnant de tout ce sang répandu, dut faire apparaître la célébration comme trop terrifiante pour ne pas être un amenuisement d'une coutume plus sauvage : si l'on acceptait alors le trépas occasionnel de jeunes gens, il fallait que celui-ci représentât une nette amélioration vis-à-vis de la situation passée. Et avec la quantité énorme de légendes de sacrifices humains, jadis adoucis en une prestation de remplacement, qui avaient cours dans toutes les contrées de l'Hellade, l'usage le plus barbare aux yeux des Grecs, le sacrifice humain, au demeurant pourvoyeur similaire de sang sur l'autel, a pu se présenter comme étant l'état antérieur le plus plausible de la fustigation.

$\mathrm{Du}$ reste, bien des légendes de sacrifices humains adoucis ressortissaient à Artémis, et il ne serait pas surprenant que le culte attique d'Halae, le premier à avoir été rapproché du mythe du retour d'Oreste, ait servi de modèle et de référence aux Spartiates : à la fin du Ve siècle, on y écorchait toujours un homme au cou pour satisfaire à l'honneur d'Artémis, et à l'époque de Théophraste, l'usage d'asperger les autels de sang humain y demeurait tenace ${ }^{28}$. L'aspersion de sang sur le $\beta \omega \mu$ ós d'Orthia, dont on pensait également la statue originaire de Tauride,

27 C'est aussi la conclusion de D. HuGUEs, Human Sacrifice (cité n. 26), p. 80.

28 Eur., Iphigénie en Tauride, 1446-1461; ThÉophraste apud Porphyre, De l'Abstinence, II, 27, 3. La ressemblance des cultes d'Orthia et de la Tauropole est grande : fondation par Oreste, statue taurique de la déesse, substitution aux sacrifices humains d'un rituel de rachat, importance terminale du sang giclant sur l'autel... L'influence de la réputation du second sur le premier n'est donc pas impossible. 
aurait, par comparaison, été également rattachée à d'antiques immolations humaines, qui n'étaient d'ailleurs, pour Philostrate, que les héritières des sinistres usages taures : pourquoi simplement cette version n'aurait-elle pas suscité la naissance d'une légende de sacrifices humains à caractères plus locaux ?

La relecture du passage de Pausanias ne semble-t-elle pas confirmer cette origine locale? Le voyageur nous signale une rixe qui naît entre les Spartiates des quatre obai, alors qu'ils sont en train de sacrifier, c'est-à-dire dans la proximité immédiate du $\beta \omega \mu o ́ s$. Le texte cependant distingue clairement deux groupes de Lacédémoniens, celui des gens de Limnatis et de Cynosouries, et celui des habitants de Mésoa et de Pitané : en bref, deux groupes qui se bagarrent à proximité de l'autel, et souillent ce dernier de sang. Ce n'est pas laisser aller son imagination que de rapprocher ces faits du texte de Xénophon, qui parle exactement des mêmes réalités, avec quelques précisions supplémentaires, à savoir le vol de fromages et les fouets.

Pourquoi dès lors ne pas tenter une tentative de reconstitution de l'évolution de la cérémonie : au Ve siècle, l'autel d'Orthia est le théâtre d'un rite pratiqué par deux groupes d'adolescents, voleurs pour les uns, fouettards pour les autres. Comme la réputation de meilleur voleur confère un renom important, les éphèbes tentent de dérober un maximum de fromages, mais par là offrent leur chair aux fouets des défenseurs et leur sang, de temps à autre, goutte sur l'autel. Pour une raison et à une époque qui nous échappent, mais avant le Ier siècle avant J.-C. et peut-être sous l'influence de cultes à pratiques similaires, dont celui d'Halae en Attique, l'épisode du vol disparut de la cérémonie tandis que la fustigation, exagérée au point de faire gicler le sang, gagnait en importance et que sa signification allait se modifiant : jadis complémentaire de l'attaque du premier groupe d'éphèbes, elle devient une épreuve d'endurance à la douleur, - comme les Gymnopédies deviennent une épreuve de résistance à la chaleur ${ }^{29}$, - et provoque parfois mort d'homme. Son caractère cruel et barbare suscite l'idée d'une origine étrangère, taurique, immanquablement liée à l'image des sacrifices humains perpétrés par Iphigénie en Tauride, tradition répercutée par Pausanias et Philostrate. Cependant, le souvenir de la lutte sanglante à l'autel reste présent, chez Plutarque par exemple, et de cette lutte est né, comme l'indique le texte du Périégète, l'aition d'une rixe entraînant effusion de sang humain sur le $\beta \omega \mu \delta \delta$, dans le sens cette

29 Voir H.I. MARRou (cité n. 13), p. 53 et 353 , n. 31. 
fois d'une impiété notoire que la divinité punit en envoyant, comme dans nombre de mythes de cet ordre, un fléau qui terrasse les survivants et en suscitant un oracle qui enjoint aux Spartiates de sacrifier des éphèbes.

En conclusion, les sacrifices humains relatifs au culte d'Orthia apparaissent plutôt comme le résultat d'interprétations et d'influences tardives sur un culte en perte de vitesse et en quête de sens. Ils ne sont donc pas originellement liés à la sphère des rites de passage, et puisqu'ils ne sont apparus, dans la littérature et les esprits, qu'à une date tardive, on ne pourra en aucun cas les tenir pour une survivance d'antiques sacrifices humains perpétrés par les Phéniciens ${ }^{30}$.

Rien n'y fait : l'établissement systématique des faits doit toujours précéder l'utilisation de ceux-ci au sein de raisonnements plus vastes, car il constitue notre seul rempart face aux envolées trompeuses de l'esprit.

Université Catholique de Louvain

Pierre BONNECHERE

Collège Érasme

Place Blaise Pascal, 1

B 1348 Louvain-la-Neuve

30 En aucun cas cette "fete aux fouets" ne peut donc traduire non plus le souvenir réel d'immolations humaines dans le culte d'Orthia (voir encore F. ROBERT, $L a$ religion grecque, Paris, 1981 [Que sais-je? 1969], p. 30), ainsi que J.G. FRAZER (Pausanias's Description of Greece, III, Londres, 1898, p. 342), pourtant peu suspect de vouloir effacer du passé grec toute trace de sacrifice humain, l'avait déjà fait remarquer, grâce à certaines comparaisons avec les initiations africaines. 